\title{
How Feasible is Extracorporeal Cardiopulmonary Resuscitation in a Medium Urban Population Centre?
}

Derek Rollo ${ }^{1}$, Paul Atkinson ${ }^{2,3}$, Jay Mekwan ${ }^{4}$, Sohrab Lutchmedial ${ }^{5}$, Joanna Middleton ${ }^{2,3}$, James French ${ }^{2,3}$, Steve Chanyi $^{6}$, James Gould ${ }^{7}$, George Kovacs ${ }^{8}$, Jean-François Légaré ${ }^{9}$, Mark Tutschka ${ }^{10}$, Jacqueline Fraser 2,3 , Michael Howlett 2,8

\begin{abstract}
1. Family Medicine, Saint John Regional Hospital, Saint John, CAN 2. Emergency Medicine, Saint John Regional Hospital, Saint John, CAN 3. Emergency Medicine, Dalhousie University, Saint John, CAN 4. Emergency Medicine, Horizon Health Network, Saint John, CAN 5. Cardiology, New Brunswick Heart Centre, Saint John Regional Hospital / Dalhousie University, Saint John, CAN 6. Cardiac/Thoracic/Vascular Surgery, Saint John Regional Hospital, Saint John, CAN 7. Emergency Medicine, Queen Elizabeth II Health Science Center / Dalhousie University, Halifax, CAN 8. Emergency Medicine, Dalhousie University, Halifax, CAN 9. Cardiac Surgery, Saint John Regional Hospital / Dalhousie University, Saint John, CAN 10. Critical Care Medicine, Saint John Regional Hospital / Dalhousie University, Saint John, CAN
\end{abstract}

Corresponding author: Paul Atkinson, paul.atkinson@dal.ca

\section{Abstract \\ Background}

Patients suffering from out-of-hospital cardiac arrest (OHCA) experience poor survival and neurological outcomes, with rates remaining relatively unchanged despite advancements. Extracorporeal membrane oxygenation (ECMO), termed extracorporeal cardiopulmonary resuscitation (ECPR) in arrests, may offer improved outcomes. We developed local screening criteria for ECPR and then estimated the frequency of use by applying those criteria retrospectively to a cardiac arrest database. The purpose was to determine if an ECPR program is feasible in a medium urban population centre in Atlantic Canada.

\section{Methods}

A three-round modified Delphi survey, building upon data from a literature review, was conducted in collaboration with external experts. The resulting selection criteria for potential ECPR candidates were applied to a pre-existing local cardiac arrest database, supplemented by health records review, identifying potential candidates eligible for ECPR.

\section{Results}

Consensus inclusion criteria included witnessed cardiac arrest, age $<70$, refractory arrest, no-flow time $<10 \mathrm{~min}$, total downtime <60min, and presumed cardiac or selected non-cardiac etiologies. Consensus

Received 11/17/2019

Review began 11/18/2019 Review ended 12/04/2019 Published 12/08/2019

๑) Copyright 2019

Rollo et al. This is an open access article distributed under the terms of the Creative Commons Attribution License CC-BY 3.0., which permits unrestricted use, distribution, and reproduction in any medium, provided the original author and source are credited. exclusion criteria were an unwitnessed arrest, asystole, and select etiologies and comorbidities. Simplified criteria were developed to facilitate emergency medical services transport. Historically, 20.0\% (95\% CI 16.2$24.3 \%$ ) of OHCA would be transported to the Emergency Department (ED), with $4.9 \%$ (95\% CI 3.0\% to $7.6 \%$ ) qualifying for ECPR.

\section{Conclusion}

Despite conservative estimates based upon historically small numbers of select cardiac arrest patients meeting eligibility for transport and initiation of ECPR, a dedicated program may be feasible in our regional hospital setting. Patient care volumes suggest it would not be resource intensive yet would be sufficiently busy to maintain competency.

Categories: Cardiac/Thoracic/Vascular Surgery, Cardiology, Emergency Medicine Keywords: cardiac arrest, resuscitation, extracorporeal membranous oxygenation

\section{Introduction}

Rates of neurologically intact survival from out of hospital cardiac arrest (OHCA) in adult patients are poor, with poor long-term outcomes [1]. The use of veno-arterial Extracorporeal Membrane Oxygenation (ECMO) in cardiac arrest, termed extracorporeal cardiopulmonary resuscitation (ECPR), may maintain vital organ perfusion, buying time for investigation and treatment of reversible causes of refractory arrest. ECPR is recognized internationally more recently as a potential tool to assist resuscitative efforts in the emergency department (ED) during cardiac arrest [2]. Observational data is suggestive that refractory OHCA treated with ECPR may lead to improved outcomes over those treated with conventional resuscitation, with survival rates as high as $48 \%$ [3]. 
Tertiary healthcare facilities based in smaller and medium urban centres that provide interventional cardiac services face the uncertainty of how feasible and sustainable the introduction of an ECPR program is in their setting. We developed site-specific screening criteria for ECPR to predict the frequency of local ECPR events and applied those criteria retrospectively to a cardiac arrest database. Results were used to determine the feasibility of an ECPR program in our small metropolitan area within Atlantic Canada.

\section{Materials And Methods}

This study was undertaken using a sequential modified Delphi and database review methodology.

\section{Determination of local ECPR selection criteria}

Published patient selection criteria currently in use for ECPR were identified by literature review [4-9]. This list formed the basis of the modified Delphi survey, involving 13 local, regional, national, and international experts, including members representing pre-hospital care, emergency medicine, cardiology, intensive care medicine, cardiac surgery, anesthesia, and perfusion technology. The team liaised with established ECPR programs in Vancouver, British Columbia, and Sydney, New South Wales. Proposed transport and team activation inclusion and exclusion criteria were scored by a panel of 13 experts. A threshold of $60 \%$ consensus was required to progress to the next round, with a final consensus rate of over $80 \%$ required to close the survey. Additional local logistical factors were also reviewed. The final New Brunswick (NB) ECPR criteria were agreed upon following three rounds and additional review by an international expert.

\section{Estimation of ECPR frequency}

A cardiac arrest database review, supplemented by a health records review for missing data, was performed following the REporting of studies Conducted using Observational Routinely-collected Data (RECORD) guidelines.

The NB-ECPR criteria were applied retrospectively to the complete database of adult patients presenting to an ED serving a medium urban population centre, in cardiac arrest, over a five-year period. Data were analyzed using standard parametric and non-parametric measures. Confidence intervals (CI) for proportions of 95\% were calculated using the modified Wald method using GraphPad Software QuickCalcs, GraphPad Software, La Jolla California USA, https://www.graphpad.com/quickcalcs/confInterval1/.

\section{Results}

\section{Modified Delphi survey}

First-round responses achieved $\geqslant 87.5 \%$ consensus for the selection of exclusion criteria. Inclusion criteria had agreement $\geqslant 62.5 \%$. Responses to the second round for inclusion criteria were unanimous at $100 \%$ with the exception of age parameters ( $<65$ years versus $<70$ years). Age was proposed to be $<70$ years following ECPR team consultation. The final set of NB-ECPR criteria for transport to hospital, and for ECPR team activation (Table 1) achieved 100\% consensus though subsequent expert review refined additional exclusion criterion based on experience (asystole). Inclusion criteria were witnessed cardiac arrest, age $<70$, refractory arrest, no-flow time $<10$ minutes, total downtime $<60$ minutes, and presumed cardiac or selected noncardiac etiologies. Exclusion criteria were unwitnessed arrest, asystole, select etiologies, and comorbidities. Simplified criteria were developed to facilitate emergency medical services (EMS) transportation. 


\section{Cureus}

\begin{tabular}{|c|c|c|c|}
\hline \multicolumn{2}{|l|}{ Criteria for Transport To Hospital } & \multicolumn{2}{|c|}{ Criteria for ECPR Team Activation } \\
\hline Inclusion Criteria & Exclusion Criteria & Inclusion Criteria & Exclusion Criteria \\
\hline & $x$ Unwitnessed Cardiac Arrest & $\checkmark$ Witnessed Cardiac Arrest & X Unwitnessed Cardiac Arrest \\
\hline & X Initial Rhythm Asystole & $\checkmark$ Age $<70$ Years Old & $x$ Asystole at Scene \\
\hline \multirow[t]{2}{*}{ Witnessed Cardiac Arrest } & $x$ Suspected Etiology: & $\checkmark$ No Flow Time $<10 \mathrm{~min}$ & $x$ Suspected Etiology: \\
\hline & -Uncontrolled Hemorrhage & $\checkmark$ Total Downtime $<60 \mathrm{~min}$ & -Uncontrolled Hemorrhage \\
\hline \multirow[t]{2}{*}{ Age $<70$ years old } & -Irreversible Brain Damage & Refractory Cardiac Arrest & -Irreversible Brain Damage \\
\hline & -Trauma & $\checkmark$ Suspected Etiology: & -Trauma \\
\hline \multirow[t]{4}{*}{ No Flow Time $<10$ minutes } & X Comorbidity: & -Cardiac & $x$ Comorbidity: \\
\hline & -Severe Disability Limiting ADLs & -Pulmonary Embolism & -Severe Disability Limiting ADLs \\
\hline & -Standing DNR Order & -Drug OD / Poisoning & -Standing DNR Order \\
\hline & -Undergoing Palliation & -Hypothermia & -Undergoing Palliation \\
\hline
\end{tabular}

\section{TABLE 1: Final NB-ECPR criteria for transport to hospital and for ECPR team activation}

NB, New Brunswick; ECPR, extracorporeal cardiopulmonary resuscitation; DNR, do not resuscitate; ADL, activities of daily living; OD, overdose

\section{Expected frequency of ECPR candidates}

Complete data were available for 273 patients presenting to the ED in cardiac arrest (see Appendix and Tables 2-3 for candidate breakdown). 


\section{Cureus}

\begin{tabular}{lc}
\hline Inclusion Criteria & Count (n) \\
\hline Witnessed Cardiac Arrest & 186 \\
Age $<70$ yo & 173 \\
No Flow <10min & 146 \\
Full Inclusion Criteria & 82 \\
Exclusion Criteria & \\
Unwitnessed Cardiac Arrest & 87 \\
\hline "Asystole" at Scene & 160 \\
\hline Suspected Etiology: & No Data \\
-Uncontrolled Bleed & No Data \\
-Irreversible Brain Damage & 10 \\
\hline -Trauma & 198 \\
Comorbidity: & 101 \\
\hline -Disability Limiting ADL & No Data \\
-Standing DNR Order & 0 \\
-Undergoing Palliation & 6 \\
\hline Total Excluded $\geq 1$ Criteria & \\
-With EMS Rhythm & \\
\hline
\end{tabular}

\section{TABLE 2: Absolute numbers of candidates meeting transport criteria}

EMS, emergency medical services; ADL, activities of daily living; DNR, do not resuscitate; ADL, activities of daily living 


\section{Cureus}

\begin{tabular}{|c|c|}
\hline Inclusion Criteria & Count (n) \\
\hline Witnessed Cardiac Arrest & 186 \\
\hline Age $<70$ years old & 173 \\
\hline No Flow < 10min & 146 \\
\hline Total Downtime $<60 \mathrm{~min}$ & 134 \\
\hline Refractory Arrest & 257 \\
\hline \multicolumn{2}{|l|}{ Suspected Etiology: } \\
\hline -Cardiac & No Data \\
\hline -Select Non-Cardiac & No Data \\
\hline Full Inclusion Criteria & 80 \\
\hline -With Downtime & 58 \\
\hline \multicolumn{2}{|l|}{ Exclusion Criteria } \\
\hline Unwitnessed Cardiac Arrest & 87 \\
\hline Asystole in ED & 189 \\
\hline \multicolumn{2}{|l|}{ Suspected Etiology: } \\
\hline -Uncontrolled Bleed & No Data \\
\hline -Irreversible Brain Damage & No Data \\
\hline -Trauma & 10 \\
\hline \multicolumn{2}{|l|}{ Comorbidity: } \\
\hline -Disability Limiting ADL & No Data \\
\hline -Standing DNR Order & 0 \\
\hline -Undergoing Palliation & 6 \\
\hline Total Excluded $\geq 1$ Criteria & 211 \\
\hline
\end{tabular}

\section{TABLE 3: Absolute numbers of candidates meeting ECPR team activation criteria}

$E D$, emergency department; ADL, activities of daily living; DNR, do not resuscitate

In all, 19 patients per year or $20.0 \%$ (95\% CI $16.2-24.3 \%$ ) of OHCA patients met eligibility for transportation to the ED. If an EMS rhythm criteria for asystole was included, only $10 \%$ (95\% CI 7.3-13.5\%) would qualify. In the ED, five patients per year, or $4.9 \%$ (95\% CI 3.0-7.6\%) would be eligible to receive ECPR. If local, inhouse cardiac catheterization hours limitations are applied, then $9.4 \%$ (95\% CI 6.8-12.9\%) would be eligible for transport from the field. For ED ECPR activation, 3.0\% (95\% CI 1.6-5.3\%) would be eligible. Further details are provided in Tables 4-5. 


\section{Cureus}

\begin{tabular}{|c|c|c|}
\hline Eligible Candidates For Transport & Total Database (\%) & Yearly count (mean) \\
\hline Without EMS Rhythm Criteria & $20.0[95 \% \mathrm{Cl} 16.2-24.3]$ & 18.5 \\
\hline With EMS Rhythm Criteria & $10.0[95 \% \mathrm{Cl} 7.3-13.5 \%]$ & 9.2 \\
\hline \multicolumn{3}{|l|}{ Considering In-House Cath Lab Hours } \\
\hline Without Rhythm & $9.4[95 \% \mathrm{Cl} 6.8-12.9]$ & 8.7 \\
\hline With Rhythm & $5.4[95 \% \mathrm{Cl} 3.5-8.2]$ & 5 \\
\hline
\end{tabular}

TABLE 4: Eligible candidates for transport by EMS

EMS, emergency medical services

\begin{tabular}{|c|c|c|}
\hline Eligible Candidates For ECPR & Total Database (\%) & Yearly Count (mean) \\
\hline Without Downtime Criteria & $4.9[95 \% \mathrm{Cl} 3.0-7.6]$ & 4.5 \\
\hline With Downtime Criteria & $3.8[95 \% \mathrm{Cl} 2.2-6.3]$ & 3.5 \\
\hline \multicolumn{3}{|c|}{ Considering In-House Cath Lab Hours } \\
\hline Without Downtime & $3.0[95 \% \mathrm{Cl} 1.6-5.3]$ & 2.7 \\
\hline With Downtime & $2.4[95 \% \mathrm{Cl} 1.2-4.6]$ & 2.3 \\
\hline
\end{tabular}

\section{TABLE 5: Eligible candidates for ECPR}

ECPR, extracorporeal cardiopulmonary resuscitation

\section{Discussion}

Despite the growing literature on ECPR practices internationally, there are no proven optimized selection criteria, and certainly none for small or medium urban centres. In this study, we have developed consensusbased criteria for ECPR and have demonstrated that the expected frequency is reasonable both in terms of skills maintenance and resource use.

Miscellaneous topics essential for the execution of a novel local ECPR program were also addressed. Mechanical CPR is an effective method for providing CPR to cardiac arrest patients and there has been evidence to show that CPR quality is greatly improved in transport while using a mechanical device [10]. Therefore, as shown in our modified Delphi, mechanical CPR devices will have a role in any future ECPR programs for OHCA. Other skills that were identified that need to be developed or sourced include rapid canalization of large blood vessels. Our Delphi showed that point-of-care ultrasound would be required for an ED-based ECPR program for vascular access and line placement.

Eligible candidates were analyzed for transport to ED by EMS, with and without the use of the EMS rhythm criterion of asystole, as historically rhythm was often categorized simply as shockable or non-shockable in the EMS data, reducing expected frequencies.

For ECPR team availability, it was agreed that initially, the program should be offered while cardiac catheterization laboratory staff are in-house. This reduces potential complicating factors of utilizing on-call resources, which can create logistical barriers and a reduction in efficiency, leading to delays. This further reduces expected candidate frequencies and hours of operation will be reevaluated during program development with the ultimate goal of unimpeded access to this service.

Finally, ECPR was never intended to be a long-term life support technique, but rather a method to extend resuscitation safely, allowing time for identifying and treating reversible causes of cardiac arrest. As such, we determined that rewarming and ECMO withdrawal should begin 24 hours post-admission, which is consistent with the existing literature [11].

\section{Limitations}


The inherent limitations of using expert opinion as a reference standard for the survey and a retrospective health records and database review are acknowledged. A contemporary literature review and liaison with international experts in the field not involved in the survey was an attempt to mitigate this. In addition, the cardiac arrest database was not designed specifically to answer this research question.

Certain conservative assumptions were applied when interpreting data. For example, patients with no cardiac arrest start time, that were witnessed arrests, were assumed to have an arrest at a time in keeping with the time of the 911 call. Total downtime was calculated using the arrival time compared with the cardiac arrest start time. Two particular limitations of the database were accounted for in the analysis. During the timeframe data was collected, all EMS in NB had automated external defibrillators, only showing shockable or non-shockable rhythms. Thus, all non-shockable rhythms were assumed asystole for secondary analysis, even though pulseless electrical activity (PEA) would be an acceptable rhythm.

In addition, we did not include patients who were not transported to hospital by EMS. Application of selection criteria to that group might impact transfer rates and subsequently impact our estimate of frequency for ECMO use.

\section{Conclusions}

Application of expert consensus-derived selection criteria for ECPR in a medium urban population centre indicates that up to one-fifth of adult out-of-hospital cardiac arrest patients would be eligible for transport to the ED for consideration of ECPR. A smaller proportion would meet the criteria for initiating ECMO in the ED. This suggests that an NB-ECPR program would not be resource-intensive yet would be sufficiently busy to maintain adequate team competency.

\section{Appendices}

\section{New Brunswick Extracorporeal Cardiopulmonary Resuscitation (NB- ECPR) Criteria Selection Survey Number 1}

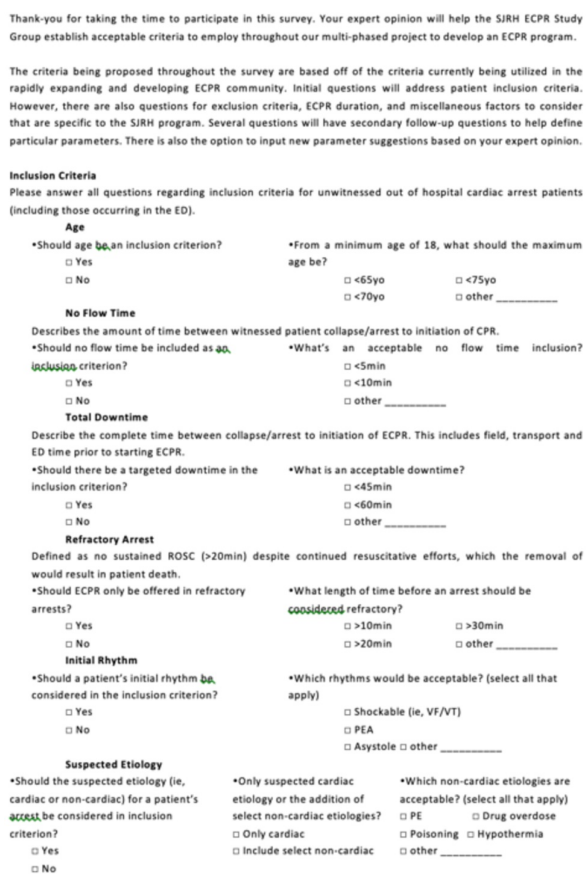

FIGURE 1: Survey 1

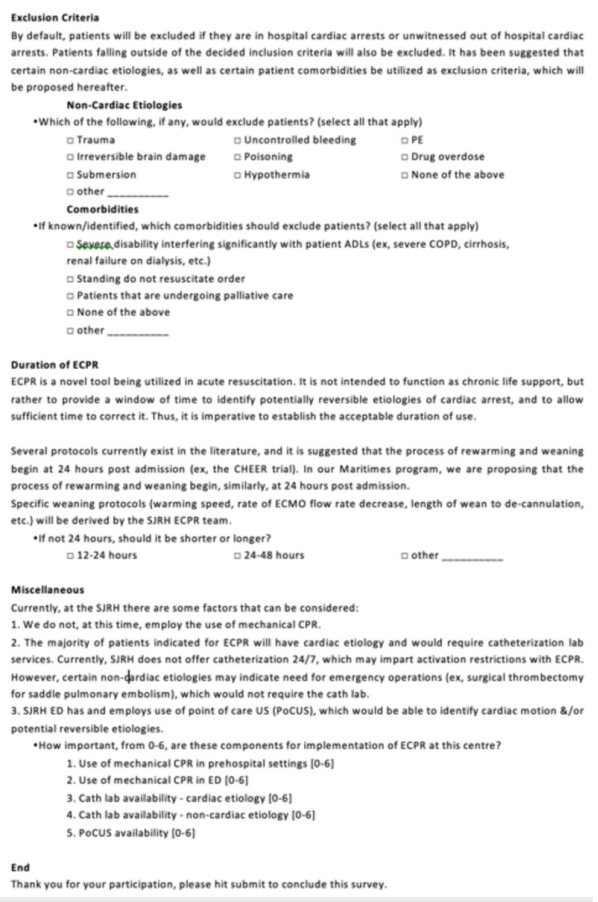

End

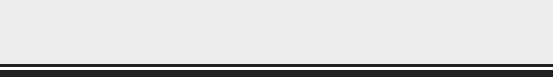

2. New Brunswick Extracorporeal Cardiopulmonary Resuscitation (NBECPR) Criteria Selection Survey Number 2 

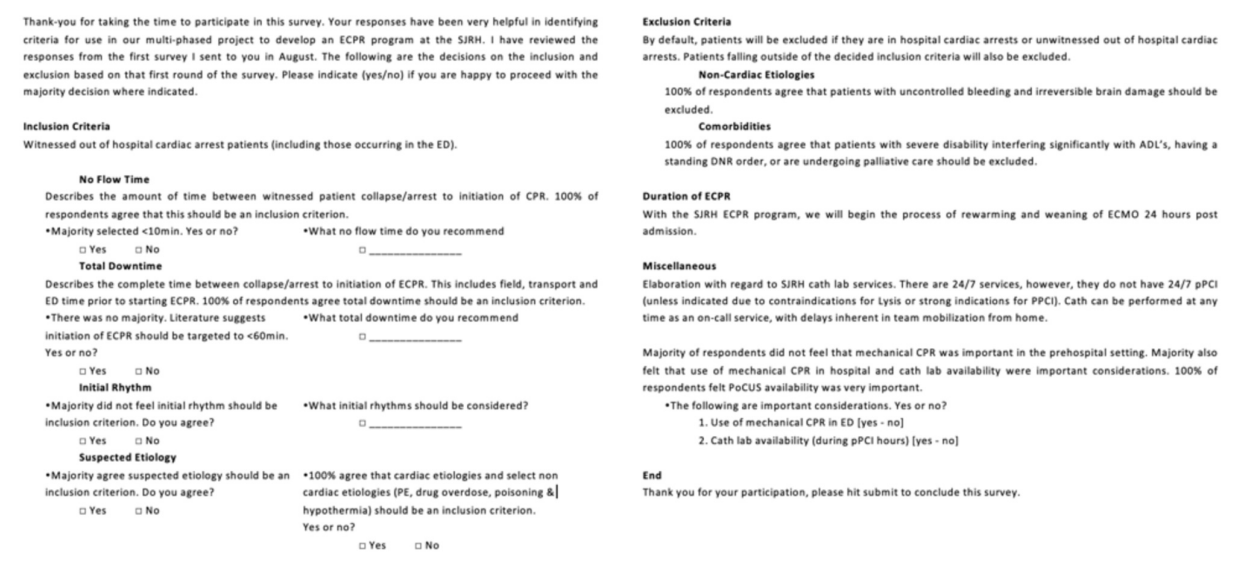

FIGURE 2: Survey 2

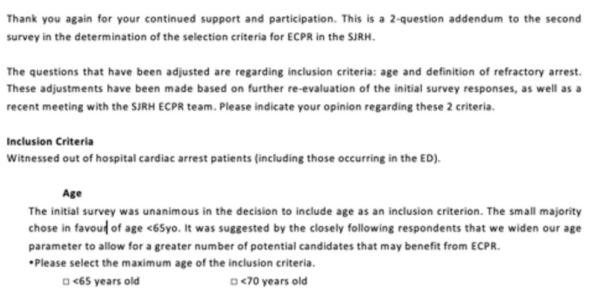

FIGURE 3: Addendum to Survey 2

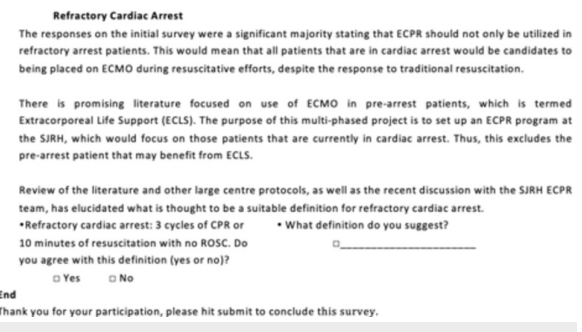

\section{Description of data extraction process}

Chart review for patients in the database with missing information included patient age, pre-arrest DNR status, terminal illness status, witnessed arrest, trauma, EMS rhythm, ED rhythm, cardiac arrest start time, time of first CPR, arrival time to hospital, and survival to admission.

After chart review, the database encompassed 395 patients. Upon review, patients who did not lose a pulse, and as such did not suffer true cardiac arrest, were excluded, leaving 371 potential candidates. Further, those with missing data points beyond the chart review were excluded from the application of criteria. There were 15 with lost charts, 15 under the age of 18,16 with a DNR, 44 because of pre-ED return of spontaneous circulation (ROSC), 3 that had ED arrests without data, 3 that were completely blank, and 2 for unknown reasons. Thus, the criteria were applied to 273 patients, while remainders were considered ineligible. Statistics and finals number of eligible candidates were compared to the inclusive database of 371 cardiac arrest ECPR candidates.

\section{Additional Information \\ Disclosures}

Human subjects: Consent was obtained by all participants in this study. Horizon Health Network Research Ethics Board issued approval 2015-2132. Ethical approval was granted by the Horizon Health Research Ethics Board (REB \#2015-2132). Animal subjects: All authors have confirmed that this study did not involve animal subjects or tissue. Conflicts of interest: In compliance with the ICMJE uniform disclosure form, all authors declare the following: Payment/services info: This project was supported by an award from the Saint John Regional Hospital Foundation. A CAN\$75,000 award was received from the Saint John Regional Hospital Foundation to the Department of Emergency Medicine Research Team to help fund the research and equipment required to implement findings from this study. The authors have no conflicts of interest to declare. . Financial relationships: All authors have declared that they have no financial relationships at present or within the previous three years with any organizations that might have an interest in the submitted work. Other relationships: All authors have declared that there are no other relationships or activities that could appear to have influenced the submitted work.

\section{Acknowledgements}


The authors are grateful to everyone who participated in the modified Delphi survey and additionally to Dr Sean Scott, NSW for his expert advice.

\section{References}

1. Kim YJ, Ahn S, Sohn CH, et al.: Long-term neurological outcomes in patients after out-of-hospital cardiac arrest. Resuscitation. 2016, 101:1-5. https://doi.org/10.1016/j.resuscitation.2016.01.004

2. Extracorporeal membrane oxygenation (ECMO) in the emergency department . (2015). Accessed: February 23, 2017: http://www.emdocs.net/extracorporeal-membrane-oxygenation-ecmo-in-the-ed/.

3. Bartos JA, Carlson K, Carlson C, et al.: Surviving refractory out-of-hospital ventricular fibrillation cardiac arrest: Critical care and extracorporeal membrane oxygenation management. Resuscitation. 2018, 132:4755. https://doi.org/10.1016/j.resuscitation.2018.08.030

4. Debaty G, Babaz V, Durand M, et al.: Prognostic factors for extracorporeal cardiopulmonary resuscitation recipients following out-of-hospital refractory cardiac arrest. A systematic review and meta-analysis. Resuscitation. 2017, 12:1-10. https://doi.org/10.1016/j.resuscitation.2016.12.011

5. Grunau B, Scheuermeyer FX, Stud D, et al.: Potential candidates for a structured Canadian ECPR program for out-of-hospital cardiac arrest. CJEM. 2016, 18:453-460. https://doi.org/10.1017/cem.2016.8

6. Kim SJ, Kim HJ, Lee HY, et al.: Comparing extracorporeal cardiopulmonary resuscitation with conventional cardiopulmonary resuscitation: a meta-analysis. Resuscitation. 2016, 103:106-116. https://doi.org/10.1016/i.resuscitation.2016.01.019

7. Ortega-Deballon I, Hornby L, Shemie SD, et al.: Extracorporeal resuscitation for refractory out-of-hospital cardiac arrest in adults: A systematic review of international practices and outcomes. Resuscitation. 2016, 101:12-20. https://doi.org/10.1016/j.resuscitation.2016.01.018

8. Ouweneel DM, Schotborgh JV, Limpens J, et al.: Extracorporeal life support during cardiac arrest and cardiogenic shock: a systematic review and meta-analysis. Intensive Care Med. 2016, 42:1922-1934. https://doi.org/10.1007/s00134-016-4536-8

9. Tonna JE, Seizman CH, Mallin MP, et al.: Development and implementation of a comprehensive, multidisciplinary emergency department extracorporeal membrane oxygenation program. Ann Emerg Med. 2017, 70:32-40. https://doi.org/10.1016/j.annemergmed.2016.10.001

10. Lyon RM, Crawford A, Crookston C, et al.: The combined use of mechanical CPR and a carry sheet to maintain quality resuscitation in out-of-hospital cardiac arrest patients during extrication and transport. Resuscitation. 2015, 93:102-106. https://doi.org/10.1016/j.resuscitation.2015.05.030

11. Kim YS, Cho YH, Sung K, et al.: Target temperature management may not improve clinical outcomes of extracorporeal cardiopulmonary resuscitation. J Intensive Care Med. 2018, 34:790-796. https://doi.org/10.1177/0885066618801269 